\title{
Pioneer Venus Multiprobe Entry Telemetry Recovery
}

\author{
R. B. Miller \\ TDA Mission Support \\ and \\ R. Ramos \\ Ames Research Center
}

\begin{abstract}
The Entry Phase of the Pioneer Venus Multiprobe Mission involved data transmission over only a two-hour span. The criticality of recovery of those two hours of data, coupled with the fact that there were no radio signals from the Probes until their arrival at Venus, dictated unique telemetry recovery approaches on the ground. The result was double redundancy, use of spectrum analyzers to aid in rapid acquisition of the signals, and development of a technique for recovery of telemetry data without the use of real-time coherent detection, which is normally employed by all other NASA planetary missions.
\end{abstract}

\section{Introduction}

Two aspects of the Pioneer Venus Multiprobe Mission dictated unique approaches to the telemetry recovery compared to other NASA planetary missions: the number of spacecraft that simultaneously transmitted data and the two-hour duration of one-chance prime data transmission. Since the four Probes entered the Venusian atmosphere essentially simultaneously, each of two large antenna ground stations that could view the entry had to be able to acquire the signal and recover the information content from four separate spacecraft simultaneously. Each Probe transmitted the data directly to Earth during the mission, which meant that only the data captured in "real-time" (i.e., as it actually happened) on Earth would ever be recovered.

The multiplicity of signals, although challenging operationally, was not a unique technological challenge since it merely required replicating the equipment ordinarily used for recover- ing telemetry data for deep space missions. See Refs. 1 and 2 for descriptions of the general problem of communications at interplanetary distances and the techniques used for NASA planetary missions. The ground equipment ordinarily used for telemetry recovery in a deep-space mission will be briefly described for completeness and to develop the framework to understand why a second method of telemetry recovery was felt to be necessary.

Fundamental to all deep space communications to date has been the process of coherent detection: first for the carrier, then the subcarrier, and finally bit synchronization. For the Pioneer Venus Multiprobe entry, a means was developed of producing a permanent record of the Probe signals as they reached the Earth without utilizing a coherent detection process. These recordings could be played after the fact many times (even backwards) into the equipment ordinarily used for telemetry recovery. In effect, the precious two hours of 
Multiprobe entry data could be repeated over and over again until all useable data had been extracted from the signals that reached the Earth.

\section{The Probes' Telecommunications Link}

The telecommunications hardware on board the Probes consisted of the formatters, multiplexers, encoders, and modulators in the Data Handling Subsystem, and the oscillators, exciters, power amplifiers, and antennas in the Communications Subsystem necessary to format and transmit the telemetry on the downlink. In addition, the Large Probe had a receiver to receive a signal from the Earth for coherency reference.

The Data Handling Subsystem received analog and digital signals from the scientific experiments and from the rest of the spacecraft. It converted the analog signals to digital data, reformatted the digital signals, and multiplexed all these data into a nonreturn-to-zero-level (NRZ-L) data stream. The data were then convolutionally encoded, and were used to phase modulate a square wave subcarrier, which was then sent to the exciter in the Communications Subsystem. The frequency of the Large Probe subcarrier was $8192 \mathrm{~Hz}$ and that of the Small Probe subcarrier was $4096 \mathrm{~Hz}$.

A block diagram of the Small Probe Communications Subsystem is shown in Fig. 1. The stable oscillator generated a signal whose frequency was approximately $19 \mathrm{MHz}$. The value was slightly different for each one of the Probes. This frequency was then multiplied by 110.5 times in the exciter to provide a carrier signal at $S$-band. The assigned frequencies of the transmitted signals are shown in Table 1.

The exciter used the subcarrier from the Data Handling Subsystem to modulate the $S$.band carrier at either 0.78 or 1.02 rad. This signal was then amplified by the driver amplifier/power amplifier to a value of $10 \mathrm{~W}$ and transmitted by a crossed dipole antenna. The antenna provided hemispherical coverage with a gain of 0 to $2 \mathrm{~dB}$ at the expected communications angles.

There was a requirement for the $S$-band signal to maintain its frequency stability to within $\pm 1 \times 10^{-9}$ from a calibration curve to provide a stable frequency standard for the Differential Long Baseline Interferometry (DLBI) Experiment.

A block diagram of the Large Probe Communications Subsystem is shown in Fig. 2. The only difference in the transmitting function between the Large Probe and the Small Probe is that the Large Probe generated $40 \mathrm{~W}$ of power by the combination of power amplifiers shown in the block diagram.
The difference in the carrier frequency generation is that the Large Probe, in addition to having the capability of generating the reference frequency by its internal auxiliary oscillator, also had the capability of receiving a signal from the ground (uplink signal) and using that signal as the reference for the downlink signal. This mode of operation is known as the coherent mode. The switch to the coherent mode was automatic anytime that the receiver was locked on the uplink signal. The modulation index used in the Large Probe was $1.18 \mathrm{rad}$.

Because the electrical power was supplied by batteries, and was therefore limited, the Probes were not turned on until 22 $\min$ before their entry into the Venus atmosphere. The Deep Space Network (DSN) stations were faced with the requirement to receive these four extremely weak signals plus the signal from the Multiprobe Bus simultaneously and extract telemetry from them during the 1.5 to $2 \mathrm{~h}$ of operation.

The total power received at the input of the low noise amplifier at the DSN stations was approximately $5 \times 10^{-19}$ to $1.6 \times 10^{-18} \mathrm{~W}$.

\section{Real-Time Coherent Detection Telemetry Recovery}

The telemetry recovery portion of the configurations used at the deep space stations is shown at a gross level in Fig. 3. An additional receiver and recording that formed a part of the DLBI wind measurement experiment is also shown. The first step in recovery is to capture as much of the energy reaching the Earth as practical, and this is accomplished with $64-\mathrm{m}$ diameter parabolic antennas. Two of the three tracking sites of NASA's DSN had Venus in view during the entry: Goldstone, CA, and Tidbinbilla, near Canberra, Australia. The first level of redundancy in data recovery was achieved by timing the entry event in the mutual view and equipping these two $64 \cdot \mathrm{m}$ sites identically for the entry event. The moving portion of the $64-\mathrm{m}$ antennas weigh $2.7 \times 10^{6} \mathrm{~kg}$ and are figured to an accuracy of $1 \mathrm{~mm}$ (rms of surface area). This 0.85 -acre surface provides an effective gain at $S$-band of $61.7 \mathrm{~dB}$, which is a factor of $1.5 \times 10^{6}$. The feed system is Cassegrainian and focuses the captured power into a low noise amplifier system located above the antenna surface. It is the signal-to-noise ratio rather than absolute signal strength that matters in communications systems. It is not possible to do anything about the external sources of noise (e.g., intergalactic, tropospheric, natural radio sources, etc.) which reach the ground antenna and undergo the same gain as the wanted signal. The critical element in achieving a useable signal-to-noise ratio is the first amplification after the antenna system. Noise is usually expressed in 
terms of the noise temperature in kelvin, and the total system noise temperature of the antenna and first amplifier system is the key figure of merit. The first amplifier used by the DSN at $S$-band is a cryogenically cooled ruby maser which adds less than $3 \mathrm{~K}$ to the noise temperature. The resulting total system noise temperature figure is only $22 \mathrm{~K}$. The maser is a broad-band device which passes all $S$-band frequencies of interest and does not have to be tuned for any particular mission.

The next step in real-time telemetry recovery is coherent phase detection and tracking of the carrier of a particular Probe. Such a phase coherent or "closed-loop" receiver must be tuned until the tuned frequency matches the received signal, then "lock" is achieved, and the closed-loop receiver will track, i.e., automatically follow, the detected carrier as the relative motion between the Probe and the Earth Doppler shifts the frequency of the signal. The locking process takes some amount of time and a skilled operator. After carrier lock is achieved, the rest of the ground system can operate at a fixed frequency $(10 \mathrm{MHz})$ and fixed signal levels because of automatic gain control in the closed-loop receiver.

The next process is subcarrier detection, another closedloop process, which takes a finite amount of time to acquire "lock." Subcarrier acquisition and tracking takes place without operator intervention because the subcarrier frequency is fully known a priori and has an essentially fixed relationship to the carrier frequency. The output of the subcarrier detection process is an integrated symbol stream. To estimate the value of individual symbols, first the bit timing has to be estimated, acquired, and then tracked. This is another closedloop tracking process that has a finite acquisition time. Quantized symbol estimates are then passed to a telemetry processing minicomputer, which must first synchronize with the telemetry frames and then decode (in the Pioneer case, using the Fano algorithm) the data. The detected and decoded (essentially error-free) data are then formatted and recorded on a magnetic tape at the tracking station. The data are also transmitted in real-time over NASA ground communications circuits to Ames Research Center. In the case of the Probes, since the entire Probe sequence took place automatically, there was no real-time control role necessary at Ames, and the flow of data to Ames was, unlike the Pioneer Venus Orbiter mission, not critical to mission success.

\section{Special Equipment to Support Real-Time Telemetry Recovery}

The above equipment was replicated four times, once for each Probe. The critical step of locking the receivers to the signals when they first appeared, and again after the large
Doppler shift of atmospheric entry, was highly dependent on operator skill. Also, a priori uncertainties in the frequencies were very large. For all previous deep space missions, the spacecraft are tracked every day prior to arriving at the planet, and the stations become very familiar with each spacecraft's characteristics. For this mission, the radio transmissions turned on just $22 \mathrm{~min}$ before hitting the atmosphere of Venus. It was not until that time that the stations and the receiver operators saw the actual probe signals. To help this situation, a means of dynamically simulating all four probe signals simultaneously, realistic both in frequency and signal level, was designed and built in-house at JPL and provided to both stations. Test and training activities took place over the 10 months preceding the entry event. In addition, to help the acquisition process, three commercial FFT spectrum analyzers were provided to each station with a switching matrix to enable each operator at each analyzer to switch to monitor any of the output of the "open-loop" receivers described below. A microprocessor was added by JPL to the commercial spectrum analyzers to allow automatic calculation of frequencies at the proper levels to pass on to the closed-loop receiver operators. The microprocessors also provide an automatic search mode for the analyzers for the eventuality of a Probe signal not appearing where expected. A $50-\mathrm{kHz}$ bandwidth was used on the spectrum analyzers in order to have enough signal-to-noise ratio per analyzer cell width to have a detectable signal. The search mode moved the $50 \mathrm{kHz}$ bandwidth automatically across the $300 \mathrm{kHz}$ bandwidth of the "openloop" receiver.

\section{Telemetry Recovery Without Coherent Detection}

The real-time telemetry recovery equipment described above had well-understood performance that could be used in the Probe telecommunications design. However, real-time telemetry recovery would require a large amount of equipment to operate properly and would be highly dependent on operator skill to assure rapid lockup of the closed-loop receivers. It was recognized, when the Multiprobe mission was in the conceptual design stage, that dependence of the mission data recovery solely on the above real-time telemetry recovery process would represent a very high risk to the mission. Based on discussions that took place in 1972, the Pioneer Venus Study Team asked DSN Engineering personnel if it might not be feasible to record the output of an open-loop receiver (a receiver set to a fixed frequency that passes everything present in a certain bandwidth), which would later be played back for telemetry recovery. In May 1973, the Study Team was informed that a precarrier detection recording did look like a feasible technology. By November 1973, based on breadboard testing, the DSN was confident enough in the approach to 
commit a performance level not to exceed 1.5-dB loss compared to the real-time telemetry recovery system. This additional loss then became a part of the mission's telecommunications design. The basic plan was then settled: enough equipment would be implemented to attempt real-time recovery for all four probes, and work would continue to engineer a means of recording the Probe signals without coherent carrier detection. The open-loop telemetry recovery configuration is also shown in Fig. 1 and involved four open-loop receivers, one per Probe. The $300-\mathrm{kHz}$ bandpass of each of these receivers encompassed all uncertainties, doppler, and useable harmonics of an individual Probe. The $300-\mathrm{kHz}$ bandpass could be set a fixed frequency for each Probe in advance of the encounter, and the only operator action required in real time was starting the recorder and changing the tape. Each $300 \mathrm{kHz}$ band was recorded on separate tracks of redundant recorders with staggered tape changes. The entry involved a total of ten receivers at each station, including the 2-MHz bandpass DLBI receiver and a contingency closed-loop receiver.

The first approach for precarrier detection recording used by DSN Engineering at JPL was to construct in-house a digital time jitter compensator to work on the output of rather antiquated analog recorders that were already in the DSN inventory. This involved recording a reference signal on one track of the analog recorder along with the spacecraft signals on separate tracks. The tape would be played back through the time jitter compensator which analog-to-digital converted a Probe signal track and the reference track, digitally servoed the Probe channel to the reference signal, and then digital-toanalog converted. This technique reduced playback phase jitter from the region of 50 to $100-\mathrm{deg}$ rms to less than 1-deg rms. The output analog signal was then upconverted back to $S$-band and inserted in front of the coherent closed-loop telemetry recovery equipment. The in-house time jitter compensator and up converter were used for the early feasibility demonstration and performance determination. With time, it became apparent that the commercial analog recording technology (involving the same kind of compensation internally) was maturing. Tests of new commercial recorders were performed that proved equal to or better than the in-house compensator used with an older commercial recorder. By March 1977, a particular commercial recorder without external compensation was selected as the final approach in concert with a JPL upconverter. Performance tests of the precarrier detection recording were now consistently getting results with less than 1-dB degradation. Although the probe entry tracking stations were provided with the necessary upconverters and interface equipment to playback the recordings for test purposes, the actual playback for the mission data would take place on DSN equipment located at JPL in Pasadena, California.

\section{Reverse Playback}

The precarrier detection recording committed to the Pioneer Venus Multiprobe mission had now assured that the entry event could be "replayed" after the fact to recover any data lost in real time due to equipment failure, operator error, or frequency uncertainties. Since the playback still involved the sequential acquisition of carrier, subcarrier, symbol stream, and decoder, there would still be a data loss, even with all time and frequency uncertainties removed a posteriori, every time the Probe signal became too weak (entry blackout, atmospheric fading, or turbulence) and during planned bit rate changes. As the precarrier detection work unfolded, the Project inquired of the DSN whether the analog recording could be played in the reverse direction. This would enable going beyond any out-of-lock condition and proceeding in the reverse direction so that the serial lockup time of the ground equipment would occur where data had already been recovered in the forward direction. The telemetry could then be recovered in the reverse direction, right up to the time of the event that forced the out-of-lock condition. After cursory study of feasibility by DSN Engineering, reverse-playback tests were performed at JPL, and negligible additional loss was measured. In 1977, the reverse-playback became part of the mission recovery plan with the DSN producing special software to digitally record the reverse symbol estimates out of the DSN equipment, and the Project taking the responsibility for decoding the reverse-playback tapes. The reverse-playback symbol decoding was performed at Ames Research Center. The technique employed was to buffer the backward frames and use the conventional forward decoding algorithm once a whole frame was collected. The software also allowed for recovery of the raw, uncorrected data for frames that could not be successfully decoded.

\section{Results}

On December 9, 1979, the sum of the Australian and Goldstone tracking stations' efforts recovered in real time an average of over 85 percent of all the data transmitted by the four probes. The combination of forward and backward playback of the precarrier detection recordings raised the recovery to essentially 100 percent of all useable data. 


\section{Note}

This article originally appeared in a special Pioneer Venus Spacecraft Instrumentation issue of the IEEE Transactions on Geoscience and Remote Sensing, Vol. GE-18, No. 1, January 1980.

\section{Acknowledgment}

A large number of DSN personnel were involved in the work described here and in the companion DLBI work described in J. Smith's article in this issue. The caliber of their work and its importance to the Mission were recognized in two NASA group achievement awards: a Pioneer Venus Multiple Data Acquisition Implementation Team award, which recognized the key personnel involved in the engineering, construction, installation, and performance verification of the systems involved, and a Pioneer Venus Ground Data System Team award, which recognized the key personnel of the DSN Operations and the deep space tracking stations, as well as the support of the DLBI experiment by Goddard Space Flight Center personnel and stations.

\section{References}

1. M. F. Easterline, "From $8-1 / 3$ bits/s to 100,000 bits/s in ten years," IEEE Commun. Soc. Magazine, Nov. 1977. (Also see related companion articles in the same issue.)

2. R. E. Edelson, et al., "Voyager telecommunications: The broadcasts from Jupiter," Science, June 1, 1979. 
Table 1. Probe frequency assignments

\begin{tabular}{ccc}
\hline Spacecraft & Uplink, MHz & Downlink, MHz \\
\hline Large Probe & 2110.317247 & 2291.747237 \\
SP1 & - & 2292.281357 \\
SP2 & - & 2292.437317 \\
SP3 & - & 2291.394837 \\
\hline
\end{tabular}

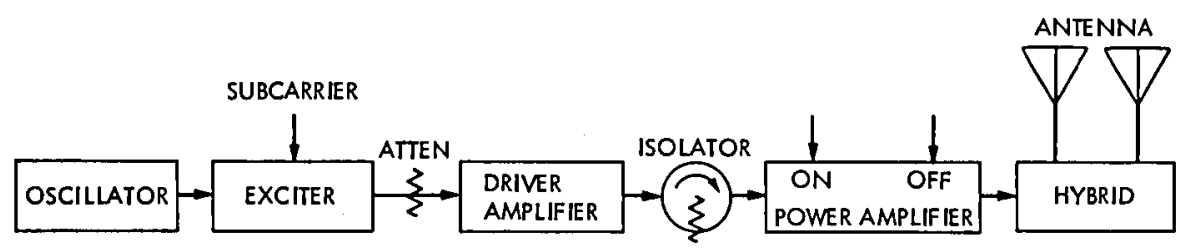

Fig. 1. Small Probe Communications Subsystem

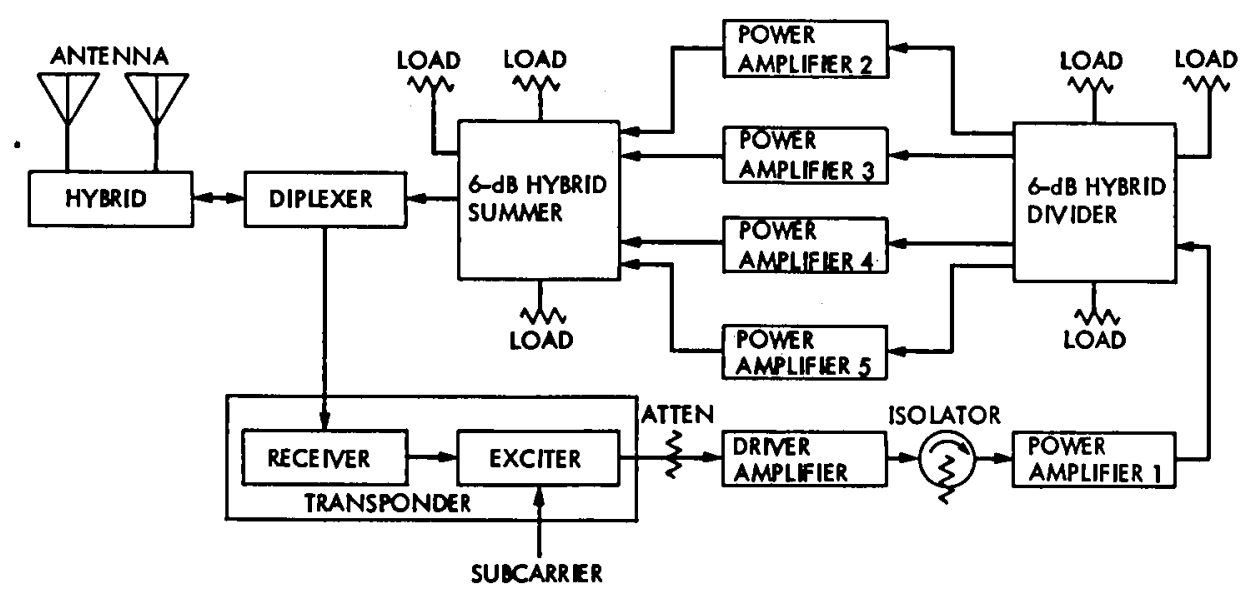

Fig. 2. Large Probe Communications Subsystem 


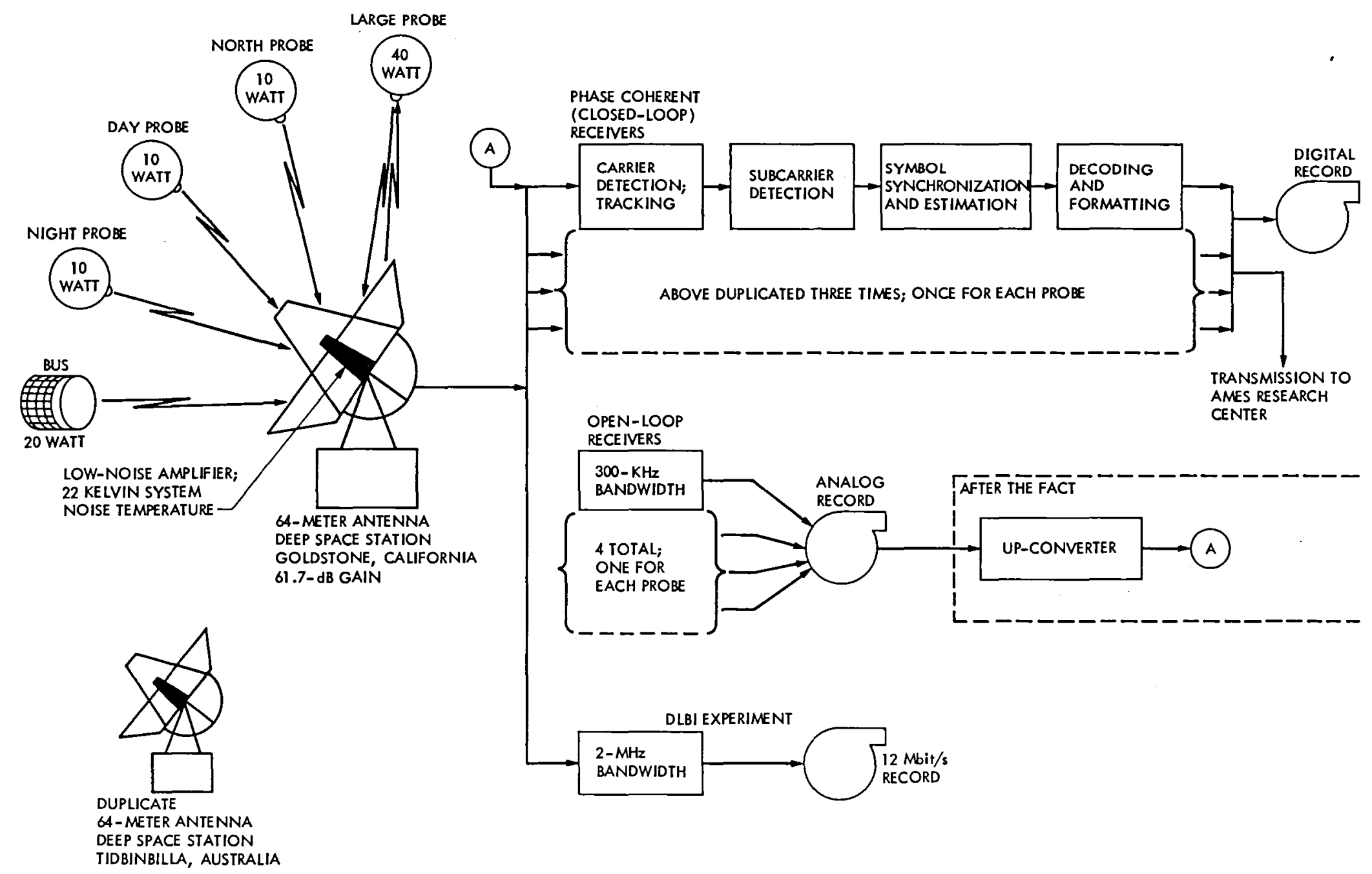

Fig. 3. Pioneer Venus Multiprobe entry data recovery configuration at the Deep Space Stations 